\title{
Can active components of licorice, glycyrrhizin and glycyrrhetinic acid, lick rheumatoid arthritis?
}

\author{
Qing-Chun Huang ${ }^{1}$, Mao-Jie Wang ${ }^{2}$, Xiu-Min Chen ${ }^{1}$, Wan-Lin Yu ${ }^{2}$, Yong-Liang Chu ${ }^{1}$, \\ Xiao-Hong $\mathrm{He}^{1}$ and Run-Yue Huang ${ }^{1}$ \\ ${ }^{1}$ Department of Rheumatology, The Second Affiliated Hospital, Guangzhou University of Chinese Medicine (Guangdong \\ Provincial Hospital of Chinese Medicine), Guangzhou, China \\ ${ }^{2}$ Central Laboratory, The Second Affiliated Hospital, Guangzhou University of Chinese Medicine (Guangdong Provincial \\ Hospital of Chinese Medicine), Guangzhou, China \\ Correspondence to: Run-Yue Huang, email: ry_huang@hotmail.com \\ Keywords: licorice, glycyrrhizin, glycyrrhetinic acid, rheumatoid arthritis, the COX-2/TXA2 pathway \\ Received: August 03, 2015 \\ Accepted: October 09, 2015 \\ Published: October 20, 2015
}

This is an open-access article distributed under the terms of the Creative Commons Attribution License, which permits unrestricted use, distribution, and reproduction in any medium, provided the original author and source are credited.

\section{ABSTRACT}

OBJECTIVES: This review stated the possible application of the active components of licorice, glycyrrhizin (GL) and glycyrrhetinic acid (GA), in rheumatoid arthritis (RA) treatment based on the cyclooxygenase (COX)-2/thromboxane A2 (TxA2) pathway.

METHODS: The extensive literature from inception to July 2015 was searched in PubMed central, and relevant reports were identified according to the purpose of this study.

RESULTS: The active components of licorice GL and GA exert the potential antiinflammatory effects through, at least in part, suppressing COX-2 and its downstream product TXA2. Additionally, the COX-2/TXA2 pathway, an auto-regulatory feedback loop, has been recently found to be a crucial mechanism underlying the pathogenesis of RA. However, TxA2 is neither the pharmacological target of non-steroidal antiinflammatory drugs (NSAIDs) nor the target of disease modifying anti-rheumatic drugs (DMARDs), and the limitations and side effects of those drugs may be, at least in part, attributable to lack of the effects on the COX-2/TXA2 pathway. Therefore, GL and GA capable of targeting this pathway hold the potential as a novel add-on therapy in therapeutic strategy, which is supported by several bench experiments.

CONCLUSIONS: The active components of licorice, GL and GA, could not only potentiate the therapeutic effects but also decrease the adverse effects of NSAIDs or DMARDs through suppressing the COX-2/TXA2 pathway during treatment course of RA.

\section{INTRODUCTION}

Because of the unwanted side effects of current drugs used for rheumatoid arthritis (RA) treatment, botanical medicines have become popular as alternative remedies as they are believed to be efficacious, safe and have over a thousand years' experience in treating patients [1]. In addition, analysis of patents on anti-RA therapies issued in China revealed that traditional Chinese Medicine may provide substantial new information for anti-RA drugs development [2]. Licorice (Glycyrrhiza glabra) is a well-known plant, which is utilized to add flavor to foods, beverages, and tobacco, and it is also used as a medicinal plant [3]. The principle component of licorice is Glycyrrhizic acid or glycyrrhizin (GL), which is a natural and major pentacyclic triterpenoid glycoside of licorice roots extracts [4] (Figure 1). GL is readily hydrolyzed to glycyrrhetinic acid (GA) in human body [5]. Following oral administration in humans as well as in rats, GL is metabolized in the gastrointestinal tract by glucuronidases into GA, which can be totally absorbed [6].

Licorice remains one of the most prescribed herbs in Chinese Medicine. There is much literature on the biological effects of the major bioactive components of licorice, particularly in terms of their anti-cancer, antiinflammatory and anti-arthritic effects $[1,5,7]$. For 
example, licorice and the roasted licorice have benefits in protecting against both acute inflammation and chronic inflammatory conditions including RA [7].

It is well known that cyclooxygenase (COX)-2 is an important target of licorice, as many constituents of licorice are able to suppress COX-2 [1, 8, 9], which is critically involved in the pathogenesis of tumor and inflammatory diseases like RA [10-13]. Five years ago, Prof. Paul M. Stewart and Stephen M. Prescott raised a question, that is, can licorice lick colon cancer?[14]. This question is raised from a discovery showing that GL reduced COX-2 activity, tumor growth, and metastasis, without the adverse effects associated with non-steroidal anti-inflammatory drugs (NSAIDs) and selective COX-
2 inhibitors (COXIBs) [15]. Today, using the same sentence pattern, we are asking the question "can active components of licorice GL and GA lick RA?" This is a data-based question, raised from several lines of evidence showing as followings. First, GL and GA provide an antiinflammatory effect by suppressing the expression and activity of COX-2 through the inhibition of nuclear factor (NF)- $\mathrm{B}$ and phosphoinositide-3-kinase (PI3K) activity [16]. Second, there are some common targets and common therapies have been revealed between RA and cancers, such as cadherin-11 and COX-2 [11, 17]. Third, COX-2 is crucially implicated in RA pathogenesis, and NSAIDs as well as COXIBs are frequently used in treating patients with RA [18]. Therefore, can active components of licorice
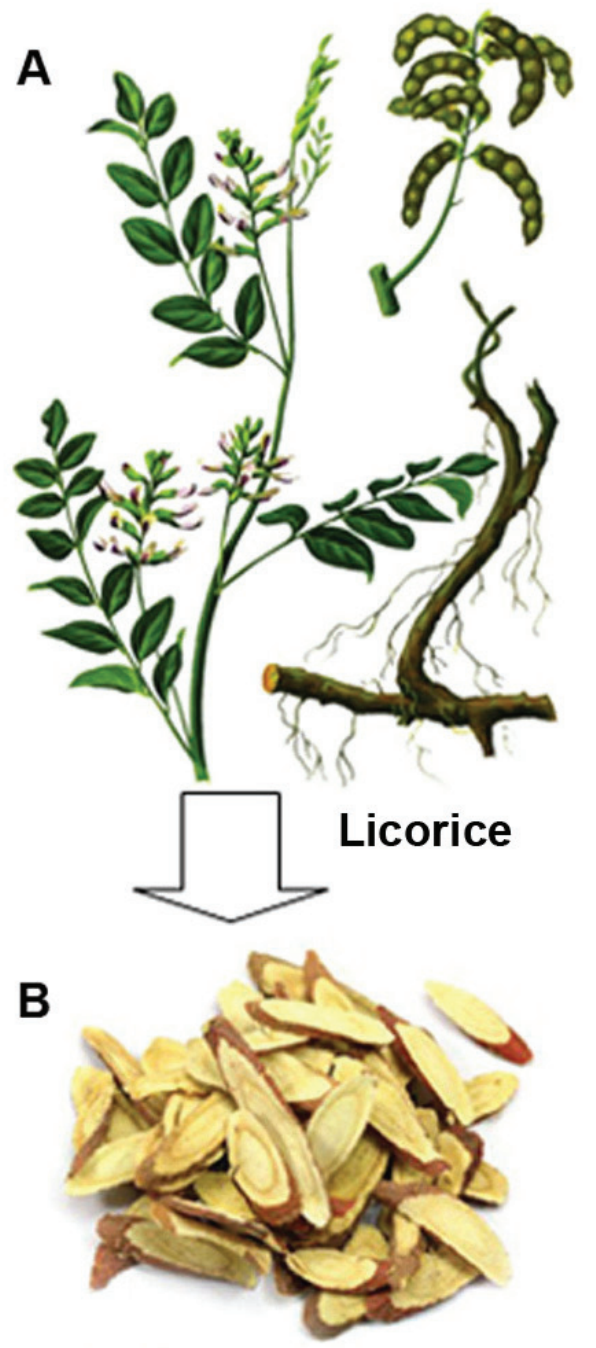

Raw licorice or the roasted licorice
used in Traditional Chinese Medicine
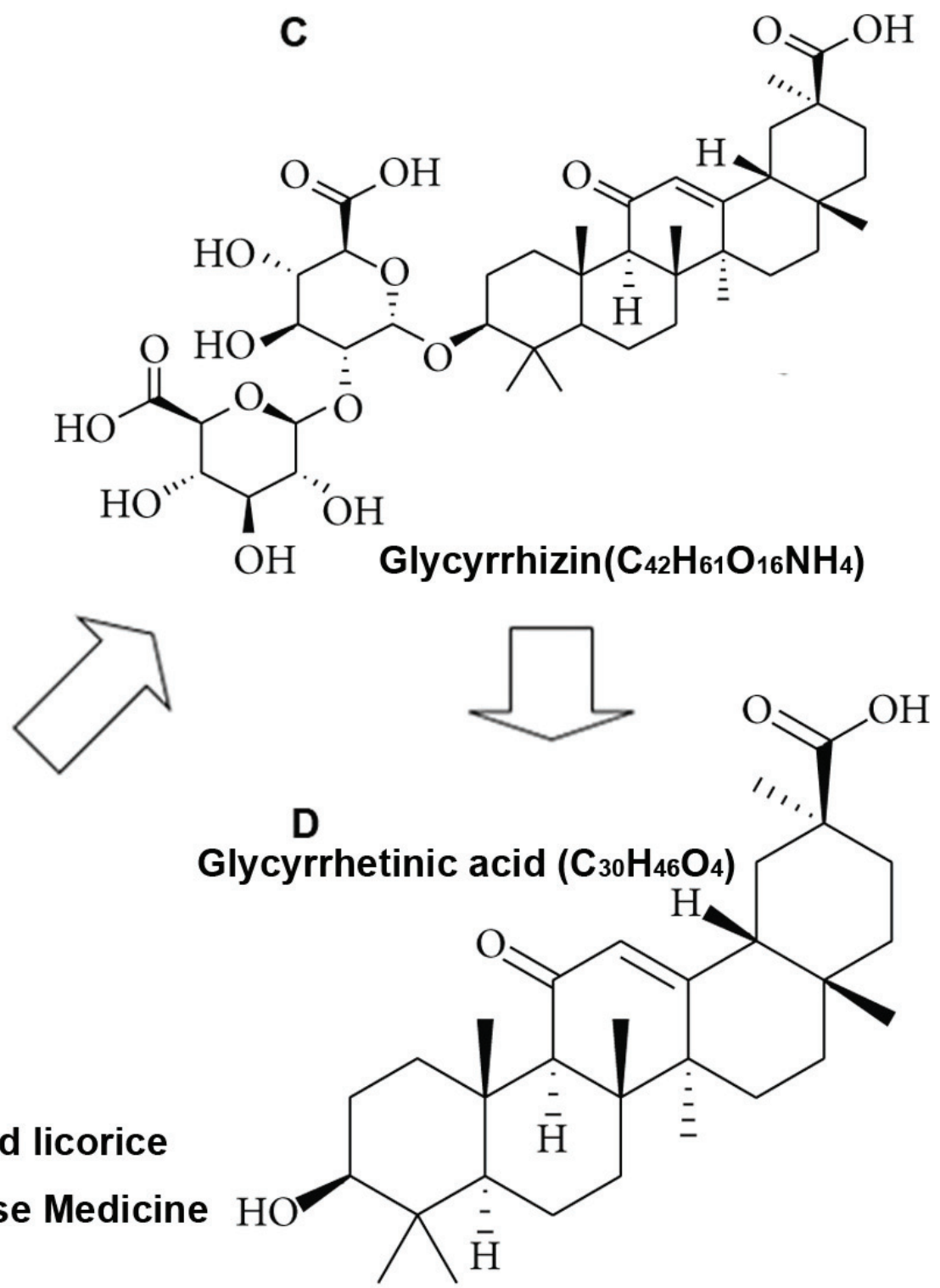

Figure 1: Licorice and its major active components. A., the Figure of licorice herb is selected from The Compendium of Materia Medica (Bencao Gangmu). B., raw licorice and the roasted licorice are frequently employed as medications in traditional Chinese Medicine. C. and D., the chemical structures of glycyrrhizin (GL) and glycyrrhetinic acid (GA). 
GL and GA lick RA? In this review, we will clarify this possibility based on the COX-2/thromboxane A2 (TxA2) pathway, which is a mechanism novelly delineated in the pathogenesis of RA $[11,19]$.

\section{METHODS}

We searched the PubMed database from inception to July 2015 with the following search terms: "licorice", "glycyrrhiza glabra", "glycyrrhizic acid", "glycyrrhizin", "glycyrrhetinic acid", "thromboxane", "cyclooxygenase-2", and/or "rheumatoid arthritis". The references within the selected reports were also considered. No limitations on language and study types. Relevant literature focusing on the field of licorice and its active components, as well as RA was identified. Three independent investigators conducted the searching process, and the experts in the field of Rheumatology were involved in the procedure of literature analysis.

\section{THE COX-2/TXA2 PATHWAY IS A PHARMACOLOGICAL TARGET OF GL AND GA}

It is well known that COX-2 is an inducible enzyme becoming abundant in inflammatory diseases including RA $[11,20]$. COX-2 catalyzes the conversion of arachidonic acid (AA) into prostaglandin $\mathrm{H} 2$ (PGH2). PGH2 is unstable and it is catalyzed by prostaglandin E synthase (PGES), prostacyclin synthase (PGIS) and thromboxane synthase (TxAS) into prostaglandin E2 (PGE2), prostacyclin (PGI) and TXA2, respectively [21, 22] (Figure 2). The role of PGES/PGE2 is to some extent controversial, as PGE2 has both pro-inflammatory and immunosuppression effects depending on cell context [22]. PGIS is generally considered to have cytoprotection effects, and the imbalance of PGI/TxA2 in favor of the latter is one of critical mechanisms underlying pathogenesis of cancer, inflammatory disease and vascular disorders [21, 22] (Figure 2). As a downstream product of COX-2 in inflammatory sites, TxA2 is a local hormone acting close to the site of its synthesis via autocrine or

\section{Licorice}

\section{Glycyrrhizin}

\section{Glycyrrhetinic acid}

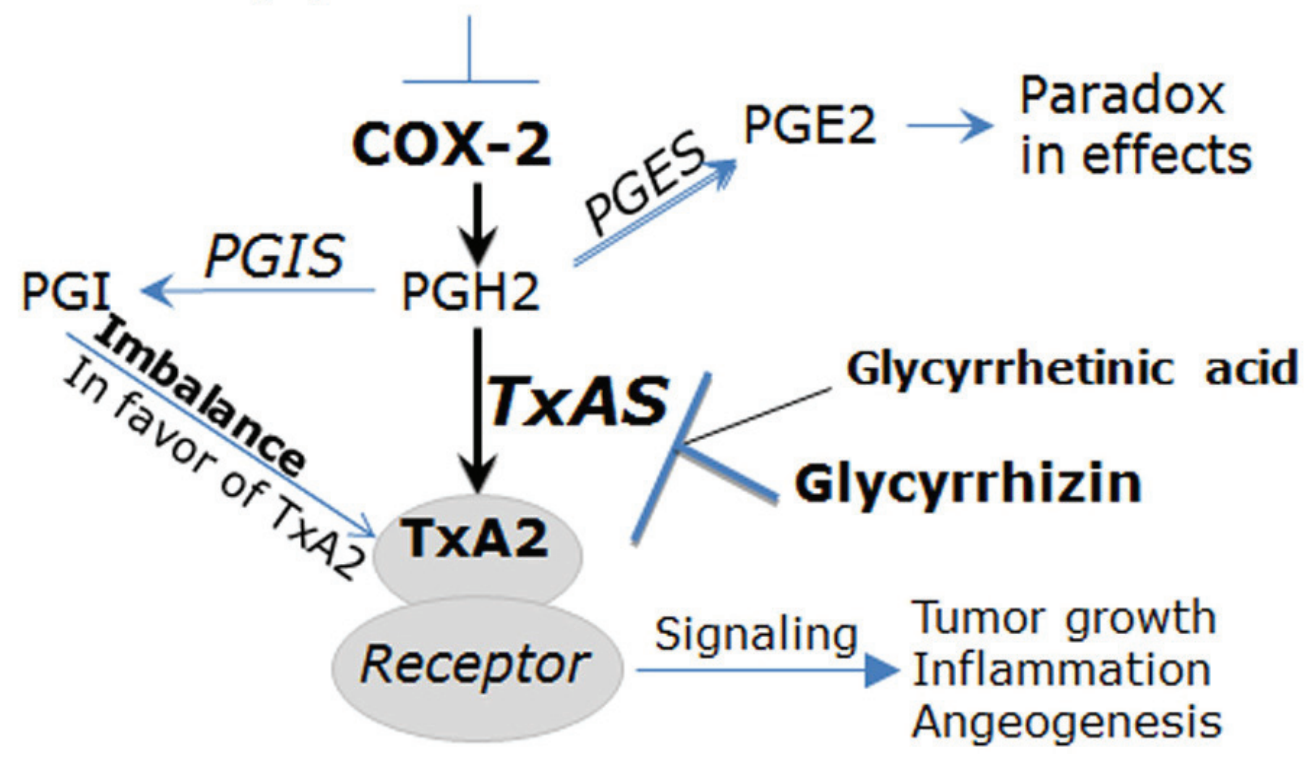

Figure 2: The COX/TxA2 pathway is the pharmacological target of glycyrrhizin (GL) and glycyrrhetinic acid (GA). Among downstream products of COX-2 pathway, PGI is generally considered to have cytoprotection effects, and the imbalance of PGI/ TxA2 in favor of the latter is one of critical mechanisms underlying pathogenesis of cancer, inflammatory disease and vascular disorders. The role of PGES/PGE2 is to some extent controversial, as PGE2 has both pro-inflammatory and immunosuppression effects depending on cell context. TxA2 acts through binding with TxA2 receptor (TP), thereby exerting promoting effects for tumor growth, inflammation and angiogenesis. Licorice and its active components GL and GA are considered to hold anti-inflammatory and anti-cancer properties through targeting the COX-2/TxA2 pathway. For example, GA inhibits lung tumor growth through suppressing expression and activity of COX-2 and TxAS and the downstream ERK/CREB signaling (Ref.5). Abbreviations: COX-2, cyclooxygenase-2; PGH2, prostaglandin H2; PGE2, prostaglandin E2; PGI, prostacyclin; TxA2, thromboxane A2; PGES, prostaglandin E synthase; PGIS, prostacyclin synthase; TxAS, thromboxane A2 synthase. 
paracrine manner [19, 21, 22]. TxA2 functions through binding with its signature receptor (Figure 2), TxA2 receptor (TP), which is a member of the G-protein-coupled cell surface receptor family $[11,22,23]$. Importantly, it is now clear that the COX-2/TxA2 pathway is an autoregulatory feedback loop for biosynthesis and action of TxA2 and it plays a key role in the pathogenesis of RA $[11,19]$.

Early studies documented that as the active component of licorice, GL is an inhibitor of COXs, thereby having anti-inflammatory and anti-tumor effects $[24,25]$. Subsequently, GL was shown to have protective effects on acute liver injury via downregulation of proinflammatory mediators including COX-2 [26]. Further study showed that GL potently protected against LPS-induced acute lung injury through, at least in part, the suppression of COX-2 [27]. These studies suggest that GL provides antiinflammatory effects with low toxicity or cytoprotective property. Several studies revealed that GL is an inhibitor of high mobility group protein B1 (HMGB1) [28], which is known to induce inflammation by enhancement of proinflammatory molecules signaling including COX-2 pathway [29]. Intriguingly, HMGB1 is expected to be a new target for RA treatment [30]. In Wister rats model of 2-acetylaminofluorene (2-AAF)-induced liver toxicity, pretreatment with GA showed potential hepatoprotective effects, which are partly attributable to the attenuation of COX-2 and its transcriptional factor NF- $\mathrm{BB}$ [31]. In human endothelial cells, the effects of TP agonist I-BOP could be mimicked by $1 \mu \mathrm{M}$ of GA with a similar time course and efficacy [32], suggesting that GA may exert its biological effects through acting on TxA2 pathway. In our laboratory, it has been found that anti-tumor effect of GL is, at least in part, TxAS-dependent [4]. Additionally, we have elucidated that, through inhibiting TxAS and its initiated extracellular signal-regulated kinas (ERK) / cAMP response element-binding protein (CREB) signaling, GA suppresses lung tumor cell proliferation [5]. It should be noted that the activities of several key molecules COX-2, TxAS and NF- $\mathrm{KB}$ were inhibited by GA, the whole effects of COX-2/TXA2 pathway is therefore suppressed by GA. Although GA may function as a TP agonist in some models, theoretically, its effects cannot be mediated through the downstream signalings, as ERK/CREB and NF- $\mathrm{kB}$ activities can be significantly inhibited by GA.

Altogether, these observations have revealed that the COX-2/TxA2 pathway is a pharmacological target of GL/GA, which provides new insights into the mechanisms of action of licorice. It also provides an explanation for the anti-inflammatory and anti-tumor effects of GL and GA, as the COX-2/TxA2 pathway is well-known to be the important molecular mechanisms underlying pathogenesis of tumor and RA [11, 19, 20, 33, 34].

\section{ROLE OF THE COX-2/TXA2 PATHWAY IN THE PATHOGENESIS OF RA}

It is well known that TxA2 is one of the downstream products of COX-2, and COX-2 as well as TxA2 are overexpressed in inflammatory conditions like RA [11, 19]. In early studies, the higher TxA2 levels were found in synovial lining obtained from RA patients, as compared to specimens from non-RA patients [35-37]. Moreover, TxA2 release was increased in peripheral blood leucocytes when cultured with RA synovial fluid exudates [38, 39]. Recently, a study recruited 54 RA patients as well as 20 healthy subjects and found that the biosynthesis of TxA2 in RA patients was significantly higher than healthy controls [40], which is in agreement with our recent report for the first time showing that serum level of TxA2 is positively correlated with 28 -joint disease activity (DAS28) score of patients with RA [19]. Additionally, we also found that in RA fibroblast-like synoviocytes (RAFLS), COX-2 effects can be mainly mediated by TxA2, and the mRNA expression of COX-2 is regulated by TxA2 action [11]. This study suggests a positive feedback for TxA2 synthesis and action in RA synovial tissue. Interestingly, it is now clear that in tumor cells, TxA2 contributes to cell proliferation through an auto-regulatory feedback loop, in which NF-кB and its downstream COX2 are involved [20]. Therefore, enlightened by these findings, we recently determined this pathway in in-vitro model of RA. The results confirmed the existence of an auto-regulatory feedback loop for TxA2 in RA FLS [19]. Through this auto-regulatory feedback loop, transcription factor NF- $\mathrm{KB}$ is activated by TxA2 signaling, COX-2 and other inflammatory factors like TNF- $\alpha$ and IL-1 are increased thereafter, thereby contributing to inflammation in RA [19] (Figure 3).

Collectively, these observations suggest that the pharmacological approaches targeting the COX-2/TxA2 pathway hold the potential as a novel add-on therapy in the treatments of RA.

\section{ADVERSE EFFECTS OF RA TREATMENT DRUGS ARE RELATED TO THE COX-2/ TXA2 PATHWAY}

Conventional disease-modifying anti-rheumatic drugs (cDMARDs) are the first-line medications used for RA treatment [41], while cDMARDs are more or less ineffective in the late phase of RA and the unwanted side effects often limit their use [42]. For example, both methotrexate (MTX) and leflunomide (LEF) are most frequently prescribed cDMARDs, while use of these two drugs entails a risk of cytopenias and the toxicity of liver and renal [41, 43]. Biologic disease-modifying antirheumatic drugs (bDMARDs) generally carry a definite increased infection and cancer risks $[41,44,45]$. 
It is now clear that, in patients with RA, TxA2 is not the molecular target of DMARDs [19]. The limitations and side effects of these drugs are considered to be, at least in part, due to lack of the effects on the biosynthesis of COX-2-derived TxA2 [11]. In support of this view, it is found that treatment of RA patients with anti-TNF- $\alpha$ agents, belonging to bDMARDs, may blunt isoprostane generation in the absence of significant effects on TxA2 biosynthesis, which could be associated with a higher frequency of non-melanoma skin cancer in patients longterm treated with anti-TNF- $\alpha$ agents $[11,40]$. In addition, MTX is found not to suppress TxA2 biosynthesis in whole blood from RA patients although MTX is a preferential COX-2 inhibitor [46]. Interesting, the combined usage of MTX and aspirin (selective TxA2 inhibitor) results in antagonism of the cytotoxic effects of MTX [47]. Therefore, these observations suggest that suppressing the biosynthesis of TxA2 that largely derived from COX-2 in inflammatory sites may produce a strong antagonistic effect to reduce the adverse effects of DMARDs.

Nonsteroidal anti-inflammatory drugs (NSAIDs) including COXIBs are routinely used for long-term therapy of RA in clinical practice $[48,49]$. However, because of the action that inhibition of COX-2-derived endothelial PGI2 without concomitant inhibition of TxA2 [50-52], some adverse effects of COXIBs, such as cardiovascular effects and renal effects are to some extent unavoidable [53-59]. Hence, the inhibitors of targeting COX-2-derived TxA2 can theoretically mitigate those adverse effects, when administered in combination with COX-2 inhibitors.

\section{GL AND GA MAY BE USED AS THE ADDITIVE TO RA TREATMENT AS THEY SUPPRESS THE COX-2/TXA2 PATHWAY}

Coupled with the observations showing the positive

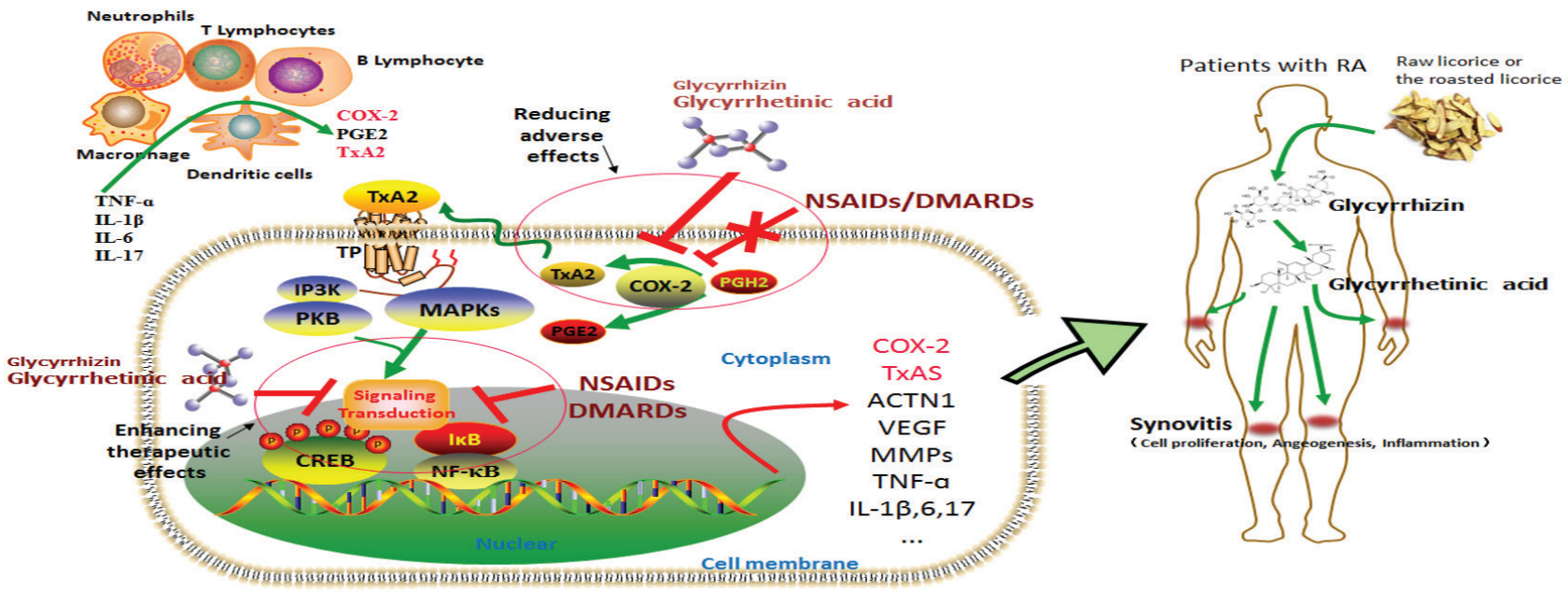

Figure 3: The COX-2/TxA2 pathway is a crucial mechanism underlying the toxicity reducing and efficacy enhancing effects of Glycyrrhizin (GL) and glycyrrhetinic acid (GA) to NSAIDs/DMARDs. We have previously elucidated a positive feedback loop for the biosynthesis and action of TxA2, i.e. the COX-2/TxA2 pathway (Ref.17). Briefly, in the inflammatory microenvironment of RA joints, lymphocytes and inflammatory cells like macrophages and neutrophils are recruited and produce many pro-inflammatory cytokines, such as TNF- $\alpha$, IL-1 $\beta$, IL-6, and IL-17 etc. By stimulation with these cytokines, the crucial molecules of COX-2 pathway, such as PGE2 and TxA2, were produced by those inflammatory cells and RA FLS. Through autocrine or paracrine signaling, TxA2 is able to specifically bind with its signature receptor TP, thereby activating several intracellular signals, MAPKs and PI3K/PKB pathways for instance. The transcription factor CREB and NF- $\mathrm{KB}$ can be subsequently activated, and thus inducing the expression of COX-2, TxAS, ACTN1, VEGF and other inflammatory cytokines. Therefore, a positive auto-regulatory feedback loop for the synthesis and action of TxA2 in inflammatory sites is formed and contributes to synovitis, a key role in pathogenesis of RA. It is thus suggested that the pharmacological approaches targeting COX-2/TxA2 pathway hold the potential as a novel add-on therapy in the treatments of RA. Both NSAIDs (including COXIBs) and DMARDs are typically prescribed medications for treatments of patients with RA. TxA2 is believed to be the non-target of NSAIDs and DMARDs. Importantly, the limitations and adverse effects of those drugs may be, at least in part, due to lack of the effects on the COX-2/TxA2 pathway. GL and GA are the major active components of licorice. Following oral administration in humans, GL is metabolized into GA in the gastrointestinal tract and GA can be totally absorbed. Fortunately, GL and GA have been reported to target the COX-2/TxA2 pathway. Therefore, GL or GA could be used as an adjunctive agent in RA treatments not only to enhance the therapeutic effects of NSAIDs/DMARDs but also to reduce the adverse effects associated with NSAIDs/DMARDs. Abbreviations: TNF, tumor necrosis factor; IL, interleukin; RA FLS, rheumatoid arthritis fibroblast-like synoviocytes; COX, cyclooxygenase; TxA2, thromboxane A2; TP, thromboxane A2 receptor; PGH2, prostaglandin H2; PGE2, prostaglandin E2; NSAIDs, non-steroidal anti-inflammatory drugs; DMARDs, disease modifying anti-rheumatic drugs; NF- $\kappa \mathrm{B}$, nuclear factor $\kappa \mathrm{B}$; CREB, cAMP response element-binding protein; MAPKs, mitogen activated protein kinases; PI3K , phosphoinositide-3-kinase; VEGF, vascular endothelial growth factor; ACTN1, $\alpha$-actinin-1; MMPs, matrix metalloproteinase. 
role of COX-2/TxA2 pathway in pathogenesis of RA, as stated above, the fact that TxA2 is not the molecular target of DMARDs and NSAIDs/COXIBs suggest that drugs targeting COX-2-derived TxA2 may reduce the negative side effects during the RA treatment course. In support of this conclusion, we have previously demonstrated that GL

A

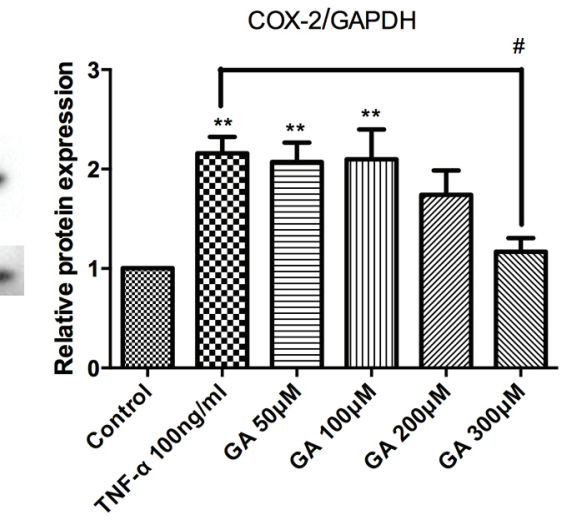

B
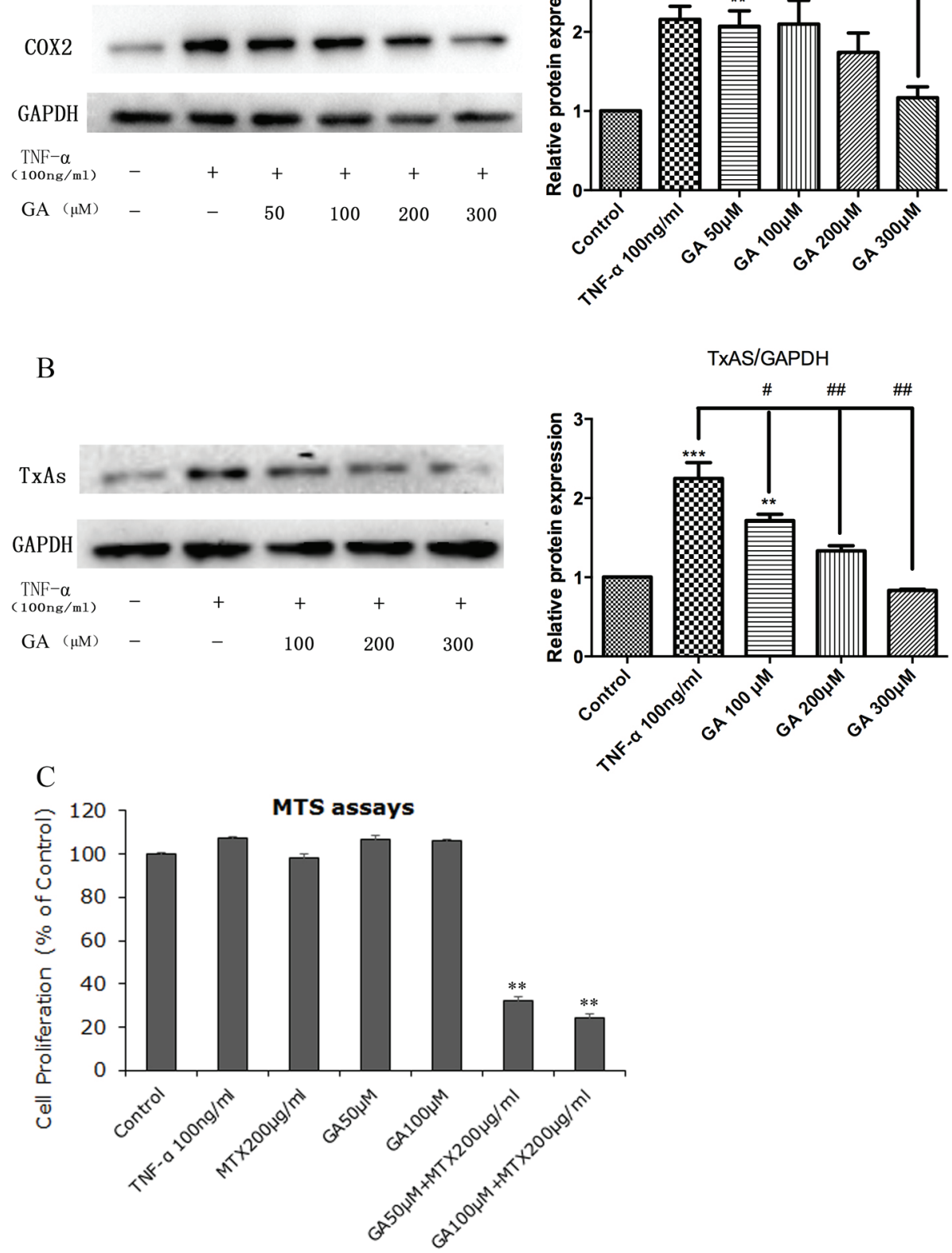

Figure 4: Effects of GA in RA FLS. Cells of RA FLS was stimulated with $100 \mathrm{ng} / \mu \mathrm{TNF}-\alpha$ for $6 \mathrm{~h}$, followed by treatment of cells with graded levels of GA for $24 \mathrm{~h}$. Cells without treatment served as controls. A. and B., GA suppressed COX-2 and TxAS expression in a dosedependent manner. The protein levels of COX-2 (72 kDa) and TxAS (60 kDa) were measured by Western blot analysis, and GAPDH (36 $\mathrm{kDa}$ ) was used as a loading control. Figure is the representative result selected from three independent experiments. Densitometry for blots was shown in the right panels. ${ }^{* *} p<0.01$ and ${ }^{* * *} p<0.001$, as compared to control; \# $p<0.05$ and \#\# $p<0.01$ as compared with TNF- $\alpha$ treatment. C., MTS assays were conducted to show the effects of GA on MTX cytotoxicity with regard to cell proliferation. There is a synergistic effect from the treatment with both GA and MTX on cell proliferation of RA FLS, suggesting GA could be used as an adjunctive agent not only to enhance the chemotherapeutic effects of MTX but also to reduce the negative side effects associated with MTX. Data are presented as percentages of the control and expressed as mean $\pm \mathrm{SD}$ of three independent experiments done in triplicate. $* * p<0.01$ when compared to control. Abbreviations: COX-2, cyclooxygenase-2; GA, glycyrrhetinic acid; MTX, methotrexate; RA FLS, rheumatoid arthritis fibroblast-like synoviocytes; TxAS, thromboxane synthase. 
and GA capable of inhibiting COX-2 expression and TxA2 biosynthesis could be used as an adjunctive agent in lung tumor not only to enhance the chemotherapeutic effects of cisplatin but also to reduce the adverse effects associated with cisplatin $[4,5]$. In a word, GL or GA can be used as an additive to treatments of RA, as they are able to target the COX-2/TxA2 pathway.

As a matter of fact, many evidences have already documented that GL or GA can be used as an adjunctive agent in treatments of RA. Triptolide, a major active component of Tripterygium wilfordii, is used for treatment in animal models of RA, whereas this natural component possesses various pharmacological activities with narrow therapeutic window and severe toxicities. In animal model studies, toxicity of triptolide can be attenuated with concomitant use of GL, as pretreatment with GL significantly accelerates the metabolic elimination of triptolide from the animal body [60]. Moreover, combined triptolide and GL treatment (triptolide $13.40 \mu \mathrm{g}$, GL 26.78 $\mathrm{mg}$ ) can reduce the arthritic index of collagen induced arthritis (CIA) rats and decrease serum levels of TNF- $\alpha$, and such effect was similar to the one measured upon application of triptolide $17.86 \mu \mathrm{g}$ [61]. It is suggested that GL can enhance the chemotherapeutic effects of triptolide. Furthermore, it has been shown that GA, MTX, and combination of GA and MTX (GA+MTX) suppressed the expression of TNF- $\alpha$ and IL-1 $\beta$ in fibroblast-like synovial (FLS) cells from CIA rats in a time-dependent manner, and the suppressing effect is GA+MTX $>$ MTX $>$ GA [62].

Altogether, the possibility of that GL and GA are utilized as the useful additive to RA treatments has been well-studied in experiments and the underlying mechanisms is, at least in part, attributable to suppression of the COX-2/TxA2 pathway. The ongoing study in our laboratory has preliminarily supported this possibility (Figure 4), and the further studies in this field are expected.

\section{CONCLUSIONS}

RA exerts profound influence on health-related quality of life, which imposed huge burdens on patients physically, mentally, and economically [63]. The general effectiveness of typically prescribed medications for patients with RA, including NSAIDs and DMARDs, has been far from satisfactory $[1,64]$. Because COX-2derived TxA2 is not the molecular target of NSAIDs and DMARDs, the limitations and negative side effects of those drugs may be, at least in part, attributable to lack of the effects on the COX-2/TxA2 pathway, which coupled with the positive role of the COX-2/TxA2 pathway in pathogenesis of RA suggests that the pharmacological approaches targeting this pathway hold the potential as a novel add-on therapy in therapeutic strategy of RA (Figure 3).

Understanding the mechanisms of action of the herbs may provide new treatment opportunities for RA patients, and the herbs used in traditional medicines provide a rich reservoir for extracting biologically active compounds. Licorice or the roasted licorice is one of the oldest and most frequently used botanicals in traditional Chinese medicine. This herb has been incorporated into recipes not only to enhance taste, but also to treat various conditions including inflammation [65]. GL and GA, active components of licorice, have been reported to target the COX-2/TxA2 pathway (Figure 3). Therefore, GL or GA could be used as an adjunctive agent in RA treatment not only to enhance the therapeutic effects of NSAIDs and DMARDs but also to reduce the adverse effects associated with NSAIDs and DMARDs (Figure 3). In a word, the COX-2/TxA2 pathway could be a crucial mechanism underlying the toxicity reducing and efficacy enhancing effects of GL and GA on typically prescribed medications, NSAIDs and DMARDs in principle, for treatments of patients with RA (Figure 3). Many researches have confirmed the toxicity reducing and efficacy enhancing effects of GL and GA on RA treatment [60-62], while further studies leading to the final application of this finding in the clinical management of RA are urgently required in the future.

\section{Abbreviations}

ACTN1, $\alpha$-actinin-1; bDMARDs, biologic diseasemodifying anti-rheumatic drugs; cDMARDs, conventional disease-modifying anti-rheumatic drugs; COXIBs, selective COX-2 inhibitors; CREB, cAMP response element-binding protein; COX-2, cyclooxygenase-2; DMARDs, disease modifying anti-rheumatic drugs; ERK, extracellular signal-regulated kinas; GA, glycyrrhetinic acid; GL, glycyrrhizin; HMGB1, high mobility group protein B1; IL, interleukin; LEF, leflunomide; MAPKs, mitogenactivatedproteinkinases; MMPs, matrix metalloproteinase; MTX, methotrexate; NF, nuclear factor; NSAIDs, non-steroidal anti-inflammatory drugs; PGE2, prostaglandin E2; PGES, prostaglandin E synthase; $\mathrm{PGH} 2$, prostaglandin $\mathrm{H} 2$; PGI, prostacyclin; PGIS, prostacyclin synthase; PI3K, phosphoinositide-3-kinase; RA, rheumatoid arthritis; TNF, tumor necrosis factor; TP, thromboxane A2 receptor; TxA2, thromboxane A2; TxAS, thromboxane synthase; VEGF, vascular endothelial growth factor.

\section{ACKNOWLEDGMENTS}

This study was supported by National Natural Science Foundation of China (No. 81302799), the joint special fund of Guangdong Provincial Department of Science and Technology-Guangdong Provincial Academy of Chinese Medical Sciences (No.2014A020221028), as well as Chinese Medical Science and Technology research funding from Guangdong Provincial Hospital of Chinese 
Medicine (No. YN2014ZH04).

\section{CONFLICTS OF INTERESTS}

There is no conflict of interests to be declared. There is no role for the funding sources in study design, data collection and analysis, data interpretation, manuscript writing, or the decision to submit this manuscript.

\section{REFERENCES}

1. Yang CL, Or TC, Ho MH and Lau AS. Scientific basis of botanical medicine as alternative remedies for rheumatoid arthritis. Clin Rev Allergy Immunol. 2013; 44:284-300.

2. Yuan HY, Zhang XL, Zhang XH, Meng L and Wei JF. Analysis of patents on anti-rheumatoid arthritis therapies issued in China. Expert Opin Ther Pat. 2015; 25:909-930.

3. Qiao X, Song W, Ji S, Wang Q, Guo DA and Ye M. Separation and characterization of phenolic compounds and triterpenoid saponins in licorice (Glycyrrhiza uralensis) using mobile phase-dependent reversedphasexreversed-phase comprehensive two-dimensional liquid chromatography coupled with mass spectrometry. J Chromatogr A. 2015; 1402:36-45.

4. Huang RY, Chu YL, Jiang ZB, Chen XM, Zhang X and Zeng X. Glycyrrhizin suppresses lung adenocarcinoma cell growth through inhibition of thromboxane synthase. Cell Physiol Biochem. 2014; 33:375-388

5. Huang RY, Chu YL, Huang QC, Chen XM, Jiang ZB, Zhang $\mathrm{X}$ and Zeng X. 18beta-Glycyrrhetinic acid suppresses cell proliferation through inhibiting thromboxane synthase in non-small cell lung cancer. PLoS One. 2014; 9:e93690.

6. Takeda S, Ishthara K, Wakui Y, Amagaya S, Maruno M, Akao T and Kobashi K. Bioavailability study of glycyrrhetic acid after oral administration of glycyrrhizin in rats; relevance to the intestinal bacterial hydrolysis. J Pharm Pharmacol. 1996; 48:902-905.

7. Kim KR, Jeong CK, Park KK, Choi JH, Park JH, Lim SS and Chung WY. Anti-inflammatory effects of licorice and roasted licorice extracts on TPA-induced acute inflammation and collagen-induced arthritis in mice. $\mathrm{J}$ Biomed Biotechnol. 2010; 2010:709378.

8. Lau GT, Ye L and Leung LK. The licorice flavonoid isoliquiritigenin suppresses phorbol ester-induced cyclooxygenase- 2 expression in the non-tumorigenic MCF10A breast cell line. Planta Med. 2010; 76:780-785.

9. Song NR, Kim JE, Park JS, Kim JR, Kang H, Lee E, Kang YG, Son JE, Seo SG, Heo YS and Lee KW. Licochalcone A, a polyphenol present in licorice, suppresses UV-induced COX-2 expression by targeting PI3K, MEK1, and B-Raf. Int J Mol Sci. 2015; 16:4453-4470.

10. Ben-Batalla I, Cubas-Cordova M, Udonta F, Wroblewski M, Waizenegger JS, Janning M, Sawall S, Gensch V, Zhao L, Martinez-Zubiaurre I, Riecken K, Fehse B, Pantel K,
Bokemeyer C and Loges S. Cyclooxygenase-2 blockade can improve efficacy of VEGF-targeting drugs. Oncotarget. 2015; 6:6341-6358. doi: 10.18632/oncotarget.3437.

11. Huang QC and Huang RY. The cyclooxygenase-2/ thromboxane A2 pathway: a bridge from rheumatoid arthritis to lung cancer? Cancer Lett. 2014; 354:28-32.

12. Shi D, Xiao X, Tian Y, Qin L, Xie F, Sun R, Wang J, Li W, Liu T, Xiao Y, Yu W, Guo W, Xiong Y, Qiu H, Kang $\mathrm{T}$, Huang $\mathrm{W}$, et al. Activating enhancer-binding protein2alpha induces cyclooxygenase- 2 expression and promotes nasopharyngeal carcinoma growth. Oncotarget. 2015; 6:5005-5021. doi: 10.18632/oncotarget.3215.

13. Hsu JY, Chang KY, Chen SH, Lee CT, Chang ST, Cheng $\mathrm{HC}$, Chang WC and Chen BK. Epidermal growth factorinduced cyclooxygenase-2 enhances head and neck squamous cell carcinoma metastasis through fibronectin upregulation. Oncotarget. 2015; 6:1723-1739. doi: 10.18632/ oncotarget. 2783.

14. Stewart PM and Prescott SM. Can licorice lick colon cancer? J Clin Invest. 2009; 119:760-763.

15. Zhang MZ, Xu J, Yao B, Yin H, Cai Q, Shrubsole MJ, Chen X, Kon V, Zheng W, Pozzi A and Harris RC. Inhibition of 11 beta-hydroxysteroid dehydrogenase type II selectively blocks the tumor COX-2 pathway and suppresses colon carcinogenesis in mice and humans. J Clin Invest. 2009; 119:876-885.

16. Wang CY, Kao TC, Lo WH and Yen GC. Glycyrrhizic acid and 18beta-glycyrrhetinic acid modulate lipopolysaccharide-induced inflammatory response by suppression of NF-kappaB through PI3K p110delta and p110gamma inhibitions. J Agric Food Chem. 2011; 59:7726-7733.

17. Assefnia S, Dakshanamurthy S, Guidry Auvil JM, Hampel C, Anastasiadis PZ, Kallakury B, Uren A, Foley DW, Brown ML, Shapiro L, Brenner M, Haigh D and Byers SW. Cadherin-11 in poor prognosis malignancies and rheumatoid arthritis: common target, common therapies. Oncotarget. 2014; 5:1458-1474. doi: 10.18632/oncotarget.1538.

18. Al-Abd AM, Al-Abbasi FA, Nofal SM, Khalifa AE, Williams RO, El-Eraky WI, Nagy AA and Abdel-Naim AB. Nimesulide improves the symptomatic and disease modifying effects of leflunomide in collagen induced arthritis. PLoS One. 2014; 9:e111843.

19. Wang MJ, Huang Y, Huang RY, Chen XM, Zhou YY, $\mathrm{Yu}$ WL, Chu YL and Huang QC. Determination of role of thromboxane A2 in rheumatoid arthritis. Discov Med. 2015; 19:23-32.

20. Huang RY, Li MY, Ng CS, Wan IY, Kong AW, Du J, Long $\mathrm{X}$, Underwood MJ, Mok TS and Chen GG. Thromboxane A2 receptor alpha promotes tumor growth through an autoregulatory feedback pathway. J Mol Cell Biol. 2013; 5:380-390.

21. Cathcart MC, Reynolds JV, O'Byrne KJ and Pidgeon GP. The role of prostacyclin synthase and thromboxane 
synthase signaling in the development and progression of cancer. Biochimica et biophysica acta. 2010; 1805:153-166.

22. Huang RY and Chen GG. Cigarette smoking, cyclooxygenase-2 pathway and cancer. Biochimica et biophysica acta. 2011; 1815:158-169.

23. Nakahata N. Thromboxane A2: physiology/ pathophysiology, cellular signal transduction and pharmacology. Pharmacol Ther. 2008; 118:18-35.

24. Craig WJ. Health-promoting properties of common herbs. Am J Clin Nutr. 1999; 70:491S-499S.

25. Wang ZY and Nixon DW. Licorice and cancer. Nutr Cancer. 2001; 39:1-11.

26. Lee CH, Park SW, Kim YS, Kang SS, Kim JA, Lee SH and Lee SM. Protective mechanism of glycyrrhizin on acute liver injury induced by carbon tetrachloride in mice. Biol Pharm Bull. 2007; 30:1898-1904.

27. Ni YF, Kuai JK, Lu ZF, Yang GD, Fu HY, Wang J, Tian F, Yan XL, Zhao YC, Wang YJ and Jiang T. Glycyrrhizin treatment is associated with attenuation of lipopolysaccharide-induced acute lung injury by inhibiting cyclooxygenase-2 and inducible nitric oxide synthase expression. J Surg Res. 2011; 165:e29-35.

28. Mollica L, De Marchis F, Spitaleri A, Dallacosta C, Pennacchini D, Zamai M, Agresti A, Trisciuoglio L, Musco $\mathrm{G}$ and Bianchi ME. Glycyrrhizin binds to high-mobility group box 1 protein and inhibits its cytokine activities. Chem Biol. 2007; 14:431-441.

29. Leclerc P, Wahamaa H, Idborg H, Jakobsson PJ, Harris HE and Korotkova M. IL-1beta/HMGB1 complexes promote The PGE2 biosynthesis pathway in synovial fibroblasts. Scand J Immunol. 2013; 77:350-360.

30. Lange SS, Mitchell DL and Vasquez KM. High mobility group protein B1 enhances DNA repair and chromatin modification after DNA damage. Proc Natl Acad Sci U S A. 2008; 105:10320-10325.

31. Hasan SK, Khan R, Ali N, Khan AQ, Rehman MU, Tahir M, Lateef A, Nafees S, Mehdi SJ, Rashid S, Shahid A and Sultana S. 18-beta Glycyrrhetinic acid alleviates 2-acetylaminofluorene-induced hepatotoxicity in Wistar rats: Role in hyperproliferation, inflammation and oxidative stress. Hum Exp Toxicol. 2015; 34:628-641.

32. Ashton AW, Yokota R, John G, Zhao S, Suadicani SO, Spray DC and Ware JA. Inhibition of endothelial cell migration, intercellular communication, and vascular tube formation by thromboxane A(2). J Biol Chem. 1999; 274:35562-35570.

33. Huang RY, Li MY, Hsin MK, Underwood MJ, Ma LT, Mok TS, Warner TD and Chen GG. 4-Methylnitrosamino1-3-pyridyl-1-butanone (NNK) promotes lung cancer cell survival by stimulating thromboxane A2 and its receptor. Oncogene. 2011; 30:106-116.

34. Huang RY, Li SS, Guo HZ, Huang Y, Zhang X, Li MY, Chen GG and Zeng X. Thromboxane A2 exerts promoting effects on cell proliferation through mediating cyclooxygenase-2 signal in lung adenocarcinoma cells. J Cancer Res Clin Oncol. 2014; 140:375-386.

35. Salmon JA, Higgs GA, Vane JR, Bitensky L, Chayen J, Henderson B and Cashman B. Synthesis of arachidonate cyclo-oxygenase products by rheumatoid and nonrheumatoid synovial lining in nonproliferative organ culture. Ann Rheum Dis. 1983; 42:36-39.

36. Blotman F, Chaintreuil J, Poubelle P, Flandre O, Crastes de Paulet A and Simon L. PGE2, PGF2 alpha, and TXB2 biosynthesis by human rheumatoid synovia. Adv Prostaglandin Thromboxane Res. 1980; 8:1705-1708.

37. Takabatake $M$, Hishinuma $T$, Suzuki N, Chiba S, Tsukamoto H, Nakamura H, Saga T, Tomioka Y, Kurose A, Sawai T and Mizugaki M. Simultaneous quantification of prostaglandins in human synovial cell-cultured medium using liquid chromatography/tandem mass spectrometry. Prostaglandins Leukot Essent Fatty Acids. 2002; 67:51-56.

38. Seitz M, Deimann W and Gemsa D. The influence of synovial fluid from patients with rheumatoid arthritis on the proliferation of peripheral blood lymphocytes and the prostanoid release from monocytes. Agents Actions. 1981; 11:606-608.

39. Seitz M and Hunstein W. Enhanced prostanoid release from monocytes of patients with rheumatoid arthritis and active systemic lupus erythematosus. Ann Rheum Dis. 1985; 44:438-445.

40. Ferrante E, Vazzana N, Santilli F, Di Cicco M, Lauriti C, Di Battista L, Ciabattoni G, Di Matteo L and Davi G. Determinants of thromboxane biosynthesis in rheumatoid arthritis: Role of RAGE and oxidant stress. Free Radic Biol Med. 2010; 49:857-864.

41. Parida JR, Misra DP, Wakhlu A and Agarwal V. Is nonbiological treatment of rheumatoid arthritis as good as biologics? World J Orthop. 2015; 6:278-283.

42. Sheikh A, Naqvi SH, Naqvi SH and Sheikh K. Itraconazole: its possible role in inhibiting angiogenesis in rheumatoid arthritis. Med Hypotheses. 2012; 79:313-314.

43. Ding CZ, Yao Y, Feng XB, Fang Y, Zhao C and Wang Y. Clinical analysis of chinese patients with rheumatoid arthritis treated with leflunomide and methotrexate combined with different dosages of glucocorticoid. Curr Ther Res Clin Exp. 2012; 73:123-133.

44. Filippini M, Bazzani C, Favalli EG, Marchesoni A, Atzeni F, Sarzi-Puttini P, Pallavicini FB, Caporali R and Gorla R. Efficacy and safety of anti-tumour necrosis factor in elderly patients with rheumatoid arthritis: an observational study. Clin Rev Allergy Immunol. 2010; 38:90-96.

45. Cush JJ and Dao KH. Malignancy risks with biologic therapies. Rheum Dis Clin North Am. 2012; 38:761-770.

46. Mello S, Barros D, Silva A, Laurindo I and Novaes G. Methotrexate as a preferential cyclooxygenase 2 inhibitor in whole blood of patients with rheumatoid arthritis. Rheumatology. 2000; 39:533-536. 
47. Yan KH, Lee LM, Hsieh MC, Yan MD, Yao CJ, Chang PY, Chen TL, Chang HY, Cheng AL, Lai GM and Chuang SE. Aspirin antagonizes the cytotoxic effect of methotrexate in lung cancer cells. Oncol Rep. 2013; 30:1497-1505.

48. Dhillon $\mathrm{N}$ and Liang K. Prevention of stroke in rheumatoid arthritis. Curr Treat Options Neurol. 2015; 17:356.

49. Tsuji S, Tomita T, Nakase T, Hamada M, Kawai $\mathrm{H}$ and Yoshikawa H. Celecoxib, a selective cyclooxygenase-2 inhibitor, reduces level of a bone resorption marker in postmenopausal women with rheumatoid arthritis. Int J Rheum Dis. 2014; 17:44-49.

50. Meagher EA. Balancing gastroprotection and cardioprotection with selective cyclo-oxygenase-2 inhibitors: clinical implications. Drug Saf. 2003; 26:913924.

51. Sanghi S, MacLaughlin EJ, Jewell CW, Chaffer S, Naus PJ, Watson LE and Dostal DE. Cyclooxygenase-2 inhibitors: a painful lesson. Cardiovasc Hematol Disord Drug Targets. 2006; 6:85-100

52. Back M, Yin L and Ingelsson E. Cyclooxygenase-2 inhibitors and cardiovascular risk in a nation-wide cohort study after the withdrawal of rofecoxib. Eur Heart J. 2012; 33:1928-1933.

53. Dessein PH, Semb AG, Gonzalez-Gay MA and Popa CD. Enhanced cardiovascular risk in rheumatoid arthritis: elucidation, assessment, and management. Biomed Res Int. 2015; 2015:850671.

54. Layton D, Souverein PC, Heerdink ER, Shakir SA and Egberts AC. Evaluation of risk profiles for gastrointestinal and cardiovascular adverse effects in nonselective NSAID and COX-2 inhibitor users: a cohort study using pharmacy dispensing data in The Netherlands. Drug Saf. 2008; 31:143-158.

55. Bansal SS, Joshi A and Bansal AK. New dosage formulations for targeted delivery of cyclo-oxygenase-2 inhibitors. Drugs Aging.2007; 24:441-451.

56. Burdan F and Korobowicz A. Coxibs: highly selective cyclooxygenase-2 inhibitors. Part II. Side effects [Article in Polish]. Pol Merkur Lekarski. 2003; 14:352-355.

57. Lai SW and Liao KF. Comparison of gastrointestinal adverse effects between cyclooxygenase- 2 inhibitors and non-selective, non-steroidal anti-inflammatory drugs plus proton pump inhibitors. J Gastroenterol. 2013; 48:674.
58. Peter D, Goggel R, Colbatzky F and Nickolaus P. Inhibition of cyclooxygenase-2 prevents adverse effects induced by phosphodiesterase type 4 inhibitors in rats. Br J Pharmacol. $2011 ; 162: 415-427$.

59. Joshi GP, Gertler R and Fricker R. Cardiovascular thromboembolic adverse effects associated with cyclooxygenase-2 selective inhibitors and nonselective antiinflammatory drugs. Anesth Analg. 2007; 105:17931804, table of contents.

60. Tai T, Huang X, Su Y, Ji J, Jiang Z and Zhang L. Glycyrrhizin accelerates the metabolism of triptolide through induction of CYP3A in rats. J Ethnopharmacol. 2014; 152:358-363.

61. Zhang W, Lu C, Liu Z, Yang D, Chen S, Cha A, Wu Z and $\mathrm{Lu} \mathrm{A}$. Therapeutic effect of combined triptolide and glycyrrhizin treatment on rats with collagen induced arthritis. Planta Med. 2007; 73:336-340.

62. Gui M1, Li Y, Wu T, Li Y, Huang Y, Zhang H. Effect of glycyrrhetinic acid on the expression of inflammatory factors in fibroblast-like synovial cells from collagen induced arthritis rats [Article in Chinese]. Xi Bao Yu Fen Zi Mian Yi Xue Za Zhi. 2012; 28:915-9.

63. Wang $\mathrm{G}, \mathrm{Mu} \mathrm{R}$ and $\mathrm{Xu} \mathrm{H}$. Management of rheumatoid arthritis in People's Republic of China - focus on tocilizumab and patient considerations. Int J Gen Med. 2015; 8:187-194.

64. Smolen JS and Aletaha D. Rheumatoid arthritis therapy reappraisal: strategies, opportunities and challenges. Nat Rev Rheumatol. 2015; 11:276-289.

65. Lee JH, Kim JE, Jang YJ, Lee CC, Lim TG, Jung SK, Lee E, Lim SS, Heo YS, Seo SG, Son JE, Kim JR, Lee CY, Lee HJ and Lee KW. Dehydroglyasperin C suppresses TPA-induced cell transformation through direct inhibition of MKK4 and PI3K. Mol Carcinog. 2015 Mar 18. doi: $10.1002 / \mathrm{mc} .22302$. [Epub ahead of print]. PMID: 25787879 . 